\title{
Anesthesia for robotic thoracic surgery
}

\author{
Henning Pauli, Mostafa Eladawy, James Park \\ Department of Cardiothoracic Anesthesia, Freeman Hospital, Newcastle Upon Tyne, UK \\ Correspondence to: Henning Pauli. Department of Cardiothoracic Anesthesia, Freeman Hospital, Freeman Road High Heaton, Newcastle Upon Tyne \\ NE7 7DN, UK. Email: henning.pauli@nuth.nhs.uk.
}

\begin{abstract}
Robotically assisted thoracic surgery (RATS) to date is performed as a small proportion of thoracic surgical procedures and only at a few centres, but is rapidly gaining more widespread interest. In this article, we present our anesthetic perspective of planning and performing RATS and highlight specific points to consider when managing these patients.
\end{abstract}

Keywords: Thoracic anesthesia; robotic surgery; thoracic surgery; human factors

Submitted Sep 01, 2018. Accepted for publication Dec 11, 2018.

doi: 10.21037/acs.2018.12.06

View this article at: http://dx.doi.org/10.21037/acs.2018.12.06

\section{Introduction}

Robotically assisted surgery is on the rise and is being increasingly utilized across a range of surgical specialties, although initially, only a few thoracic centres developed a robotic surgery program. After publications of initial experiences (1-4) and amidst the ongoing debates of the benefits (5), an increasing number of thoracic centres are adding robotically assisted thoracic surgery (RATS) to their portfolio. The range of procedures performed includes cardiac operations, oesophagectomies, lung resections, diaphragm repairs and resection of mediastinal tumours.

By volume of procedures carried out worldwide, the Da Vinci Robotic Surgical System (Intuitive Surgical Inc. Sunnyvale, CA, USA) is the most commonly used platform.

It provides three-dimensional (3D) video imaging, plus a set of telemanipulated flexible effector instruments (6). The system consists of three major components: a console for the operating surgeon, a patient-side cart with four interactive robotic arms, and a vision cart including optical devices for the robotic camera. In this article, we will focus on the anesthetic implications of RATS procedures carried out at our institution using the Da Vinci system, which are lobectomies, thymectomies, wedge biopsies and diaphragmatic repairs.

\section{Starting a RATS program}

The preparation and execution of robotic thoracic surgery involves a large team, including surgeons, scrub nurses, anesthetists and anesthetic assistants. Ideally, the team initially consists of the same regular members to minimize the learning curve. All members of the team have to undergo sufficient training to be familiar with the equipment, specific problems and bailout procedures. The process involves specific surgical training, visits to an established centre, careful patient selection, procedure planning, team discussion and eventually the first procedure under supervision of an experienced proctor (7). Advanced planning and rehearsal should determine designated roles, theatre layout, required equipment, backup and bailout procedures. While these are all defined in the procedure planning, it is recommended to confirm these before the start of the case in the form of a team briefing or group hug.

\section{Anesthetic management}

The core anesthetic skillset required for RATS is the same as is for open thoracic surgery. Experience in lung isolation techniques, one lung ventilation and related complication management is a prerequisite.

\section{Pre-assessment}

The selection and pre-assessment for patients undergoing robotic thoracic surgery follows the same principles as for open or VAT surgical procedures. The aim is to 
stratify perioperative risk to be balanced against the proposed benefit. For lung resections, there are five key areas reflecting patient risk: cardiovascular, respiratory, age, performance and nutritional status. The authors follow the British Thoracic Society guidelines in terms of surgical candidate selection and fitness for proposed surgery (8). Minimally invasive surgery is often described as a component of an enhanced recovery (ER) program. ER integrates a bundle of measures aiming to decrease perioperative stress and expediting patient recovery. These measures consist of preparation for surgery through patient assessment and education, minimal starvation times and carbohydrate loading, minimally invasive surgery, optimal fluid management and pain control, and the rapid reintroduction of oral nutrition and mobilisation following surgery (9).

With a reasonable body of evidence for colorectal surgery and increasingly for some other specialities, it would seem intuitive to commence the RATS patient group on such a pathway. Established programs report quicker recovery times and reduced analgesia requirements, which suggest less of a surgical insult; however, robotic surgery may have longer procedural times and carries a noteworthy conversion rate to open surgery (10). Consequently, it is our opinion that a patient not fit for open surgery is also not a candidate for robotic surgery. Furthermore, patients with borderline lung function may not tolerate one lung ventilation for prolonged periods of time and an open procedure would possibly be more feasible for these patients utilizing intermittent, nondependent lung ventilation which surgically can be easier dealt with in the course of an open procedure. Additionally, other than the deleterious effects of $\mathrm{CO}_{2}$ insufflation in RATS on gas exchange, the cardiovascular compromise may also not be tolerated in some patients and arguably some of these patients may be able to tolerate an open procedure. We generally do not use sedative premedication, unless there are specific patient factors. Analgesic or analgesia-sparing premedication (e.g., Gabapentin) could be considered.

\section{Monitoring}

In addition to routine monitoring [i.e., electrocardiogram (ECG), pulse oximetry, temperature, $\mathrm{ETCO}_{2}$ and spirometry] an arterial line is placed for beat-to-beat blood pressure monitoring and regular blood gas analysis. After the initial learning curve with potentially prolonged procedural times, we no longer use central venous access routinely, unless there is significant cardiac or renal comorbidity. The CVP will rise during $\mathrm{CO}_{2}$ insufflation, but the clinical value and utility of this observation in a patient in lateral position on one lung ventilation is uncertain. If placed, it should be inserted on the operative side to avoid the risk of pneumothorax to the dependant lung. Equally, we avoid inserting urinary catheters routinely as it is no longer in keeping with the philosophy of ER.

\section{Anesthetic technique}

The specific anesthetic techniques and drugs used should follow the local practice; both total intravenous anesthesia (TIVA) and volatile anesthesia are suitable. Equally, the choice of lung isolation technique should be according to local preference and expertise. In our centre, we use double lumen tubes (DLT) whenever possible. The sidedness of the DLT remains a topic of discussion amongst thoracic anesthetists. While it is technically possible and generally more popular to use left-sided DLTs in the vast majority of cases, our practice is to routinely use a DLT to the opposite side of surgery. In any case, bronchoscopic confirmation of optimal device placement is highly recommended before the patient is draped, as manipulation perioperatively is difficult due to poor access with some models. For this reason, a bronchial blocker would be our second choice, as we feel that these may potentially require more frequent readjustments. In general, once correctly placed, we have not experienced many problems with device migration, as movement and retraction in the surgical field is minimal during RATS compared to open thoracotomy.

The effects of anesthesia, patient position, surgical manipulation and one lung ventilation on ventilation and perfusion requires an understanding of these changes and experience in troubleshooting any resulting hypoxia. $\mathrm{CO}_{2}$ insufflation will add to gas exchange challenges by increasing airway pressures, thus reducing tidal volumes to the dependent lung. Additionally, $\mathrm{CO}_{2}$ diffusion contributes to $\mathrm{PaCO}_{2}$ rise and acidosis, potentially aggravated by its effects on haemodynamics. We recommend a protective lung ventilation strategy and allowing permissive hypercapnia to a $\mathrm{pH}>7.25$ as long as this is haemodynamically tolerated and the patient has no comorbidities precluding an elevated $\mathrm{PaCO}_{2}$. We use PEEP, limit Paw to a maximum of $30 \mathrm{cmH}_{2} \mathrm{O}$ and avoid high respiratory rates or shortened $\mathrm{I}: \mathrm{E}$ ratios, as this may cause air trapping in this patient group who often suffer from a degree of chronic obstructive pulmonary disease (COPD). 
Fluid therapy remains a debated issue in thoracic surgery and should be according to the local practice for this patient group. In our centre, we follow a relatively restrictive one. Blood loss is generally small, but $\mathrm{CO}_{2}$ insufflation may cause haemodynamic instability due to compression of mediastinal vessels. This reduction in venous return may require a volume bolus in combination with vasoconstrictors, bearing in mind the restrictive fluid strategy. However, large bore access should always be placed for the eventuality of a major haemorrhage, which requires the capacity to infuse large volumes of warmed fluids and readily available cross matched red cells. Temperature monitoring and the diligent use of patient and fluid warming devices are important.

Special attention should be placed on adequate muscle relaxation throughout the robotic procedure, as patient movement or coughing may cause tissue to be torn or damaged. As the robot does not transmit tissue tension or movement to the operator, tissue grasped by the robotic instruments will not be released in response to patient movement. Therefore, patient coughing may result in tissue injury and potentially serious bleeding. Strong patient movement or coughing may also cause the robot to stop, displaying an error message and requiring a reboot to continue surgery. Therefore, we recommend neuromuscular monitoring and consideration of continuous infusion of a suitable muscle relaxant, such as Atracurium.

\section{Pain control}

In our experience, the analgesic requirements to control acute pain in RATS is reduced. Like in conventional thoracic procedures at our hospital, we use a combination of paravertebral block after induction, surgically placed paravertebral catheter and opioid PCA. This is supplemented by simple analgesics and Gabapentin for 3 days. Generally, we tend to avoid NSAIDs immediately postoperatively, due to concerns over bleeding and renal injury. Intraoperative opioid doses are reduced compared to open or VATS procedures and remifentanil can be used during surgery to control response to surgical stimulus while avoiding the sedative effects of a larger, long-acting opioid bolus. While in some centres epidural analgesia for thoracic surgery remains popular, we feel that it is not warranted in RATS. Chronic post-thoracotomy pain is reported in a significant number of VATS and in even more (25-60\%) open patients (11). This is thought to be due to nerve trauma from retraction during thoracotomy or levering in the intercostal space during VATS. This type of chronic pain can lead to a slower recovery, and the need for chronic use of neuropathic analgesia.

It has been suggested that this trauma is reduced due to the pivoting of the robotic arms around the entry point in the chest wall rather than levering or pressing. The incidence of this observed in our series was only 6\% (12). Further reduction in pain may be achieved by a sub-xiphoid approach for lobectomy to deliver often significantly airtrapped lung specimens. Using this approach, rather than an anterior utility port through an intercostal space, we observed improved pain control (12). Reduced postoperative pain aids quicker recovery, mobilization and discharge home. Like in any other thoracic surgery, we aim to extubate the patient at the end of the procedure. In line with other thoracic surgery patients, after a recovery period in PACU, the patient can be transferred to an HDU or suitably equipped and staffed ward depending on the local setup. Improved pain control facilitates patient progression as per ER with early establishment of oral diet and mobilization.

\section{Specific issues relating to robotic surgery are: patient position}

Precise patient positioning is important in enabling optimal robot access and docking. At the same time, care has to be taken to prevent pressure damage, nerve injury (13) or crushing injuries as the robot's powerful arms can exert high force without haptic feedback to the operating surgeon. For a robotic lung resection and for diaphragmatic procedures, the patient is placed in a maximally flexed lateral decubitus position, similar to a nephrectomy position. The table break is at mid thorax to open up the rib spaces and a cushion may be placed under the patient at the level of the umbilicus to attain a horizontal line of shoulder, ribs, flank and hips. A beanbag is useful for patient positioning and a hip strap is applied to secure and maintain patient position. Care should be taken to protect pressure points with gel or foam pads. Particular care has to be taken to position the arm to avoid overextension or pressure giving rise to brachial plexus injury. It is also advisable to maintain a slight headup position to avoid head and upper airway congestion.

For mediastinal surgery, the patient is positioned supine with the side of surgical access at the edge of the table. Choice of side for access is dependent on operator preference, but the right side may be the easier approach early on in the learning curve. The arm is suspended below the plane of the body against the edge of the table and the 
right hemi-thorax is elevated with a long gel roll or sand bag placed underneath the patient just below the scapula. The robot comes in over the opposite shoulder to the side of surgery. A slight reverse Trendelenburg position helps the mediastinum and diaphragm fall away from the neck region.

\section{Patient access}

For the anesthetic team, patient access to the patient can be limited and problematic. In lung resections, the Da Vinci $\mathrm{Si}$ and $\mathrm{X}$ dock over the patient's head, leaving only a small area of access for the anesthetist in the sterile surgical field. It is crucial that the patient faces the anesthetic team and anesthetic machine in case of need for airway intervention. The theatre layout has to be adjusted, either by moving the anesthetic machine or by arranging the robotic cart accordingly.

The Da Vinci Xi can be positioned at the side of the patient, making access to the head somewhat easier but still relatively limited.

When the robot is docked, limited access to the patient mandates meticulous attention to lung isolation, monitor and line placement to minimize having to intervene or readjust near the patient. The use of long anesthetic tubes, sufficient length monitoring leads and extensions on intravenous and monitoring lines with position of transducers away from the patient is recommended. Transparent drapes at the patient head end are useful, as they offer visualization of the airway and vascular access devices and assessment of skin colour.

\section{$\mathrm{CO}_{2}$ insufflation}

Similar to laparoscopic procedures, $\mathrm{CO}_{2}$ insufflation with flow rates of $5-10$ litres/min is used in RATS. This raises intrapleural pressure to $5-10 \mathrm{mmHg}$ and aids in expanding the surgical field and pushing the diaphragm towards the abdomen. The negative effects for the anesthetist to be prepared for are haemodynamic instability, due to compression of mediastinal vessels, leading to hypotension and bradycardia. This may require adequate volume therapy, vasoconstrictors, anticholinergic drugs and sometimes reduction or cessation of $\mathrm{CO}_{2}$ insufflation. Our local practice is to limit $\mathrm{CO}_{2}$ insufflation to exert a maximum pressure of $8 \mathrm{mmHg}$.

$\mathrm{CO}_{2}$ insufflation also may cause hypercarbia, which may be difficult to control during one lung ventilation. As mentioned before, we recommend a protective lung ventilation strategy, allowing permissive hypercapnia, rather than trying to maintain normocarbia with aggressive ventilation, provided this is haemodynamically tolerated and the patient has no co-morbidities precluding elevated $\mathrm{PaCO}_{2}$. Thirdly, insufflation of cold $\mathrm{CO}_{2}$ poses challenges to maintaining normothermia in often long procedures. Lastly, $\mathrm{CO}_{2}$ insufflation, giving rise to high intrathoracic pressures, carries the potential risk of gas embolism, which has multiple publications in laparoscopic surgery. While not yet reported in RATS, it has been described in other types of robotic surgery (14) and we would advise vigilance and consideration as differential diagnoses in sudden haemodynamic collapse.

\section{Hemodynamic instability}

Hypotension and arrhythmias more commonly occur due to manipulation or compression of cardiac structures by the robotic instruments. The subxiphoid positioning of a trocar and extraction of the lung specimen, in particular, frequently cause haemodynamic compromise. Observing the surgical steps on the vision monitor easily allows recognising the cause of the instability and alerting the surgeon to release tissue tension or move the instruments to allow for prompt recovery. Furthermore, particularly at the start of a program and early in the operator learning curve, the lack of haptic feedback (the ability to feel the softness of tissues, and the resistance they offer to the surgeon's movements) may be the cause of inadvertent tissue injury and haemorrhage. Significant injury may also result from moving an instrument outside the field of vision, because the immense power of the robotic arms may tear through any structure in its path without giving tactile feedback of an obstruction to the operator. The first indication of major vascular structure injury may be the loss of vision due to bleeding, followed by rapid hemodynamic collapse.

\section{Communication and human factors}

Due to the amount and nature of the equipment, the operator is somewhat isolated from the patient side, surgical assistant and anesthetic team. With his head "inside" the console and immersed in $3 \mathrm{D}$ vision, there is the risk of task fixation, losing track of time and tunnel vision. While the head is engaged in the console, communication from the operator is via a loudspeaker transmitted through a microphone. Often the sound quality is surprisingly poor, particularly given the otherwise high-end specification 
and cost of the equipment. This potentially hampered communication has to be recognized and clear pathways should be in place to establish clear communication. In practice, the operator head has to come out of the console for face-to-face communication in case of procedural difficulties, patient instability or lack of progress.

\section{Bailout procedure}

A number of situations require conversion to a Plan $\mathrm{B}$, the open thoracotomy.

It is important for the team to have an established and rehearsed drill for rapid undocking and removal of the robot out of the surgical field. Thoracotomy tray, sternotomy tray and saw, cross-matched blood, resuscitation equipment and drugs should be readily available. Conversion may become necessary in cases of slow progress due to technical or anatomical difficulties, poor visualisation or exposure, persistent bleeding, poor lung isolation or the patient not tolerating the haemodynamic or respiratory challenges of the lengthy procedure. This may be required as an emergency in case of uncontrolled haemorrhage, severe haemodynamic instability or cardiac arrest. In the event of an uncontrollable haemorrhage, the bleeding point is compressed with a swab on a ring clamp if possible (15). All robotic instruments are removed from the arms, the robot is undocked and robot cart is wheeled away. The camera is kept in its port and held by an assistant as in a VATS procedure, visualizing the bleeding area and aiding compression with the swab whilst the surgeon performs the thoracotomy to control the bleeding. In case of cardiac arrest, the patient should be moved to a supine position to allow for effective delivery of advanced life support and consideration of a sternotomy.

\section{Conclusions}

RATS is gaining popularity and might compete with conventional VATS procedures in many centers in the near future. As rewards for embarking on a new procedure with an inevitable learning curve, we have seen shorter hospital stay, reduced postoperative pain and high levels of patient satisfaction in our patient group. Although the anesthetic management of RATS is based on the core practice of thoracic anesthesia, some details are distinctly different and requires special attention. Understanding the principles of managing anesthesia for robotic procedures, including caveats and complications, is paramount in achieving a successful operation and satisfactory patient experience.

\section{Acknowledgements}

None.

\section{Footnote}

Conflicts of Interest: The authors have no conflicts of interest to declare.

\section{References}

1. Melfi FM, Menconi GF, Mariani AM, et al. Early experience with robotic technology for thoracoscopic surgery. Eur J Cardiothorac Surg 2002;21:864-8.

2. Bodner J, Wykypiel H, Wetscher G, et al. First experiences with the da Vinci operating robot in thoracic surgery. Eur J Cardiothorac Surg 2004;25:844-51.

3. Park BJ, Flores RM, Rusch VW. Robotic assistance for video-assisted thoracic surgical lobectomy: technique and initial results. J Thorac Cardiovasc Surg 2006;131:54-9.

4. Rea F, Marulli G, Bortolotti L, et al. Experience with the "da Vinci" robotic system for thymectomy in patients with myasthenia gravis: report of 33 cases. Ann Thorac Surg 2006;81:455-9.

5. Ricciardi S, Cardillo G, Zirafa CC, et al. Robotic lobectomies: when and why? J Vis Surg 2017;3:112 .

6. Mack MJ. Minimally invasive and robotic surgery. JAMA 2001;285:568-72.

7. Cerfolio RJ, Bryant AS, Minnich DJ. Starting a robotic program in general thoracic surgery: why, how, and lessons learned. Ann Thorac Surg 2011;91:1729-36; discussion 1736-7.

8. Lim E, Baldwin D, Beckles M, et al. Guidelines on the radical management of patients with lung cancer. Thorax 2010;65 Suppl 3:iii1-27.

9. Simpson JC, Moonesinghe SR, Grocott MP, et al. Enhanced recovery from surgery in the UK: an audit of the enhanced recovery partnership programme 2009-2012. Br J Anaesth 2015;115:560-8.

10. Cao C, Manganas C, Ang SC, et al. A systematic review and meta-analysis on pulmonary resections by robotic video-assisted thoracic surgery. Ann Cardiothorac Surg 2012;1:3-10.

11. Wildgaard K, Ravn J, Kehlet H. Chronic postthoracotomy pain: a critical review of pathogenic mechanisms and strategies for prevention. Eur J 
Cardiothorac Surg 2009;36:170-80.

12. Society for Cardiothoracic Surgery in Great Britain and Ireland. Perspectives In Cardiothoracic Surgery. Available online: https://www.ctsnet.org/sites/default/files/images/ ION\%20VOL\%202\%20Web.compressed.pdf

13. Pandey R, Elakkumanan LB, Garg R, et al. Brachial plexus injury after robotic-assisted thoracoscopic thymectomy. J Cardiothorac Vasc Anesth 2009;23:584-6.

Cite this article as: Pauli H, Eladawy M, Park J. Anesthesia for robotic thoracic surgery. Ann Cardiothorac Surg 2019;8(2):263268. doi: $10.21037 /$ acs.2018.12.06
14. Hong JY, Kim JY, Choi YD, et al. Incidence of venous gas embolism during robotic-assisted laparoscopic radical prostatectomy is lower than that during radical retropubic prostatectomy. Br J Anaesth 2010;105:777-81.

15. Campos J. Anesthesia for Robotic Thoracic Surgery. In: Slinger P. Editor. Principles and Practice of Anesthesia for Thoracic Surgery. New York, NY: Springer, 2011;445-51. 